\title{
Caracterização Isotópica de Metabasitos e Anfibolitos dos Grupos Açungui e Setuva na Porção Sul da Faixa Ribeira
}

\author{
Marcos Aurélio Farias de O liveira ${ }^{1}$, Vanderlei Maniesi ${ }^{2}$, Wilson Teixeira ${ }^{3}$, Elias Carneiro Daitx ${ }^{1}$ \\ ${ }^{1}$ Departamento de Petrologia e Metalogenia - Instituto de Geociências e Ciências Exatas - UNESP \\ Caixa Postal 178, CEP 13506-900, Rio Claro, SP, BRA \\ 2Universidade Federal de Rondônia - UNIR, Porto Velho, RO, BRA \\ ${ }^{3}$ Centro de Pesquisas G eocronológicas - Instituto de Geociências - USP, São Paulo, SP, BRA
}

Palavras-chave: metabasitos e anfibolitos, isótopos, geocronologia, Faixa Ribeira, contaminação crustal.

\begin{abstract}
RESUMO
Análises isotópicas de $\mathrm{Pb}, \mathrm{Rb}, \mathrm{Sr}$, Sm e Nd foram realizadas em anfibolitos de Campo Largo (PR), Rio Branco do Sul (PR) e Adrianópolis (PR) e em metabásicas de Adrianópolis e Apiaí (SP), pertencentes aos Grupos Açungui e Setuva, porção sul da Faixa Ribeira. Essas ocorrências foram escolhidas por que já contam com estudos geoquímicos pormenorizados, que apontam para fontes mantélicas com características peculiares para cada uma delas. Os únicos resultados da literatura referentes à idade desses corpos são do metagabro de Apiaí, com valores neoproterozóicos de $617 \pm 4 \mathrm{Ma}(\mathrm{U} / \mathrm{Pb}$ em zircão), e de $839 \pm 85 \mathrm{Ma}(\mathrm{Rb} / \mathrm{Sr}$ em rocha total). Os dados isotópicos obtidos permitiram calcular idades $\mathrm{Sm} / \mathrm{Nd}$ por isócrona mineral, usando rocha total, plagioclásio e piroxênio, respectivamente de $820 \pm 84$ Ma para a metabásica de Adrianópolis e de $885 \pm 53$ Ma para o metagabro de Apiaí. Esses resultados, que coincidem aproximadamente com a idade do metagabro de Apiaí, obtida pelo método $\mathrm{Rb} / \mathrm{Sr}$, estão sendo tentativamente interpretados como uma estimativa da época de extração do manto do magma gerador dessas rochas, com base em valor positivo de $\varepsilon_{\mathrm{Nd}(\mathrm{t}) \text {, obtido em amostra de }}$ Adrianópolis. Valores de $\varepsilon_{\mathrm{Nd}(0)}$ plotados contra a razão $\left({ }^{87} \mathrm{Sr} /{ }^{86} \mathrm{Sr}\right){ }_{0}$ mostram que, os pontos correspondentes aos diversos corpos estudados, distribuem-se em posições diferentes. A maioria das amostras segue aproximadamente a tendência do "mantle array", a partir do MORB, em direção ao manto enriquecido e/ou domínio de contaminação crustal, sendo a metabásica de Adrianópolis a mais primitiva e o anfibolito, também de Adrianópolis, o mais enriquecido. Pode-se dizer assim, que as assinaturas isotópicas distintas entre os corpos ora estudados sugerem uma derivação a partir de diferentes fontes mantélicas, como já evidenciado pelos dados geoquímicos. Valores negativos de $\varepsilon_{\mathrm{Nd}}$ e elevados da razão ${ }^{87} \mathrm{Sr} /{ }^{86} \mathrm{Sr}$, para algumas amostras, deixam claro, no entanto, que parte desse material registra modificações causadas por processos metamórficos, de contaminação crustal e/ou de alteração.
\end{abstract}

Keywords: matabasite and amphibolite, isotopes, geochronology, Ribeira Belt, crustal contamination.

\begin{abstract}
$\mathrm{Pb}, \mathrm{Rb}, \mathrm{Sr}, \mathrm{Sm}$ and $\mathrm{Nd}$ isotope analyses were carried out on amphibolitic rocks from Campo Largo (PR), Rio Branco do Sul (PR) and Adrianópolis (PR) and for the metabasite from Adrianópolis and Apiaí (SP), all belonging to the Açungui and Setuva Groups, southern portion of the Ribeira Belt. These occurrences were chosen because each exhibits geochemical data indicative of a mantle source, having different signatures. Geochronologic determinations are available only for the Apiaí metagabbro with Neoproterozoic ages of $617 \pm 4 \mathrm{Ma}(\mathrm{U} / \mathrm{Pb}$ in zircon) and $839 \pm 85 \mathrm{Ma}(\mathrm{Rb} / \mathrm{Sr}$, whole rock). Age determinations by $\mathrm{Sm} / \mathrm{Nd}$ mineral isochron using whole rock, plagioclase and pyroxene yield values of $885 \pm 53 \mathrm{Ma}$ for the Apiaí metagabbro and $820 \pm 84 \mathrm{Ma}$ for the Adrianópolis metabasite. These results agree within error with the $\mathrm{Rb} / \mathrm{Sr}$ isochron of $839 \pm 85$ Ma may possibly be interpreted as the time of extraction of the magma from the mantle, as suggested

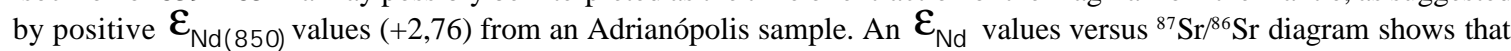
each studied occurrence plots in a different position, following approximately the mantle array trend, the most primitive being the metabasite of Adrianópolis, near the MORB field, and the most enriched being the amphibolite from the same region, near the enriched mantle and or crustal contamination field. The distinct difference in isotopic signatures between the amphibolite and metabasite suggests derivation from different mantle sources, as supported by available geochemical data. Nevertheless, some of the samples show isotopic evidence of the action of metamorphism, crustal contamination and weathering.
\end{abstract}




\section{IN TRO DU ÇÃO}

A porção sul da Faixa Ribeira é caracterizada pela presença de espessos pacotes de rochas metassedimentares, com baixo a médio grau metamórfico, contendo numerosas ocorrências de rochas metabásicas e anfibolitos que constituem os Grupos Açungui e Setuva, nos estados do Paraná e São Paulo (Almeida, 1956; Bigarella \& Salamuni, 1956; Ebert, 1971; Pontes, 1982; Fritzson Junior et al., 1982; Hasui et al., 1984; Fiori, 1985; 1987; 1990; Soares, 1987). Várias dessas ocorrências de rochas metabásicas vêm sendo caracterizadas petrologicamente, revelando serem ortoderivadas, com caráter de toleito oceânico enriquecido, formadas em ambiente de bacia retro-arco (Maniesi, 1997; Campanha, 1991; Maniesi \& Oliveira, 2000; Maniesi \& Oliveira, 2002).

Partindo-se destes conhecimentos sobre as rochas metabásicas e anfibolitos, foram realizados estudos isotópicos para uma caracterização petrogenética. As determinações ( $\mathrm{Rb}, \mathrm{Sr}, \mathrm{Pb}, \mathrm{Sm}$ e $\mathrm{Nd}$ ) foram efetuadas no Centro de Pesquisas Geocronológicas do Instituto de Geociências da Universidade de São Paulo.

\section{SITUAÇÃO GEO LÓ GICA E CARACTERÍSTICAS PETRO G RÁFICAS}

Os Grupos Açungui e Setuva fazem parte da extremidade sul da Faixa Ribeira de Almeida et al. (1973) ou mais especificamente da Faixa de Dobramentos Apiaí de Hasui et al. (1975) e Hasui et al. (1980). Outras denominações são conhecidas da literatura tais como Cinturão de Dobramentos Apiaí de Campos Neto \& Figueiredo (1995) ou Domínio Apiaí de Campanha \& Sadowski (1999), e definem a situação específica da porção sul, onde pacotes de rochas supracrustais de baixo grau estão melhor preservados, em contraste com a porção norte, que apresenta grau metamórfico mais elevado.

O Grupo Açungui é composto por metassedimentos de baixo grau metamórfico, representados por várias associações contendo metarenitos, metapelitos, metaritmitos, metacalcários e metadolomitos, provenientes de diversos sistemas deposicionais, incluindo fluviais, litorâneos, de plataformas carbonáticas e terrígenas e dispostos estruturalmente em três sinclinórios: Capiru, Votuverava e Itaiacoca, sendo o ambiente tectônico dessa unidade o de bacia tipo retro-arco, originado provavelmente no
Neoproterozóico (Soares, 1987; Fiori, 1985, 1987, 1993; Campanha, 1991; Campanha \& Sadowski, 1999; Hackspacher et al., 2000).

Já o Grupo Setuva é constituído por associações metassedimentares e metavulcano-sedimentares com metarenitos, metapelitos, metacalcários manganesíferos, metatufos, vulcânicas andesíticas a basálticas e metachert ferrífero entre outras, originados em bacia tipo retro-arco, no mesoproterozóico (Soares, 1987; 1988). Estruturalmente essas rochas exibem padrão de dobramento isoclinal fechado, relacionado a cisalhamento dúctil de baixo ângulo (Pontes, 1982; Soares, 1987). A presença de associações minerais com biotita, granada e estaurolita indica metamorfismo de grau médio para essas rochas (Piekarz, 1981; Reis Neto \& Soares, 1987).

O estudo isotópico foi desenvolvido em amostras de metabásicas e anfibolitos das regiões de Apiaí, SP, Adrianópolis, PR e Rio Branco do Sul, PR, pertencentes ao Grupo Açungui e Campo Largo, PR, associada ao Grupo Setuva, ocorrências essas já caracterizadas por estudos geoquímicos detalhados (Maniesi, 1997; Maniesi \& Oliveira, 2000; Maniesi \& Oliveira, 2002, Figura 1).

Estudos geoquímicos realizados nas quatro ocorrências ora descritas indicam que geneticamente, de um modo geral, elas estão relacionadas à olivina toleitos subalcalinos com assinaturas de basalto oceânicos, tipo MORB ou EMORB, destacando-se os tipos de Rio Branco do Sul pelo caráter mais magnesiano, empobrecimento em elementos incompatíveis e afinidade com basalto komatiítico. A análise desse comportamento é feita com utilização de diagramas de elementos incompatíveis, de ETR e diagramas multielementos e revela que essas rochas originaram-se de fontes diferentes, pois as assinaturas nos "spidergrams" são características e peculiares para cada uma delas (Maniesi, 1997; Maniesi \& Oliveira, 2000; Maniesi \& Oliveira, 2002). Em adição são características também as anomalias positivas, principalmente de $\mathrm{Rb}$ e por vezes de $\mathrm{Ba}, \mathrm{Nb}$, Th e Sm e negativas de $\mathrm{K}$, Ta e $\mathrm{Zr}$, estas últimas principalmente para a ocorrência de Rio Branco do Sul. A natureza dessas anomalias, no entanto, é difícil de precisar, podendo estar ligada a processo de contaminação crustal.

A ocorrência de Apiaí (metagabro de Apiaí) constitui um corpo de $30 \mathrm{~km}$ de extensão por 1,5 a 2,5 km de largura, intrusivo em metassedimentos da porção superior do Grupo Açungui (metamargas, metacalcários e metapelitos), onde, por vezes, produz auréolas de contato. É representada por rochas constituídas essencialmente pelos minerais augita, hiperstênio, plagioclásio (An 50-70) e hornblenda castanha, classificando-se assim como 


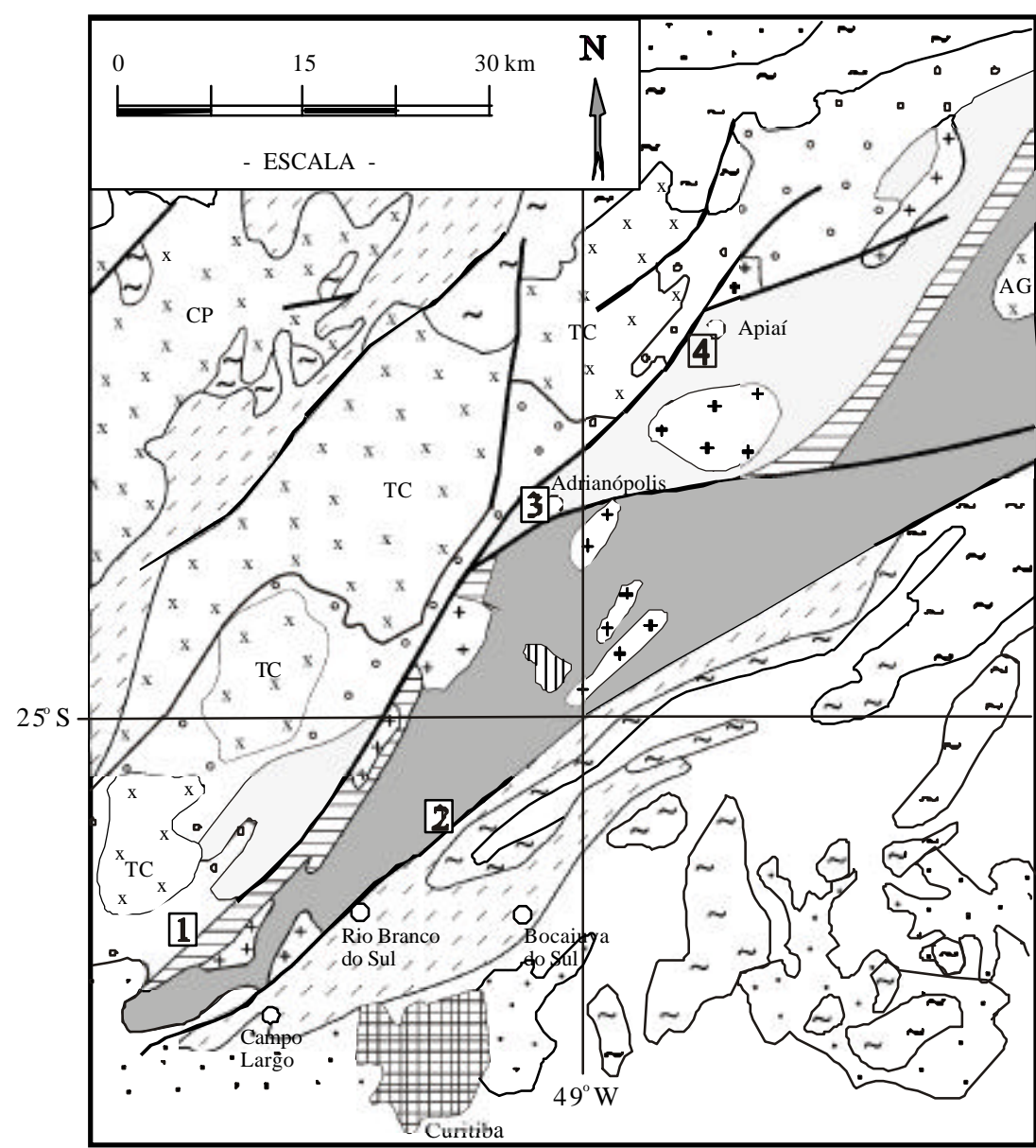

\section{LEGENDA}

$\because$ Coberturas fanerozóicas e cenozóicas

Rochas intrusivas alcalinas.

+++ Granitóides.

Complexos granitóides; CP- Cunhaporanga TC - Três Córrego; AG - Agudos Grandes.

Dolomitos e carbonatos. Seqüência de águas rasas litorâneas, baixo grau metamórfico.

Metabrecha, metaconglomerado, metapelitos.

Sequiência vulcano-sedimentar, com domínio de rochas carbonatadas. Médio e baixo grau metamórfico.

Carbonatos e rochas psamo-pelíticas. Carbonatos de águas rasas plataformais não litorâneas de baixo grau matamórfico.

Seqüência vulcano-sedimentar com domínio de rochas psamo-pelíticas, médio e baixo grau metamórfico.

Gnaisses variados, xistos e quartzitos de médio a baixo grau metamórfico.

Gnaisses e migmatitos variados. Alto a médio grau metamórfico.

1 = Área de Campo Largo; 2 = Área de Rio Branco do Sul; 3 = Área de Adrianópolis; 4 = Área de Apiaí.

Figura 1. Distribuição dos litotipos do sul de São Paulo e parte do Paraná (retirado de Campanha et al., 1987), com localização das áreas estudadas.

gabronorito ou localmente como leucogabronorito (Daitx et al., 1990; Campanha, 1991; Maniesi et al., 1999). Modificações metamórficas são vistas ao longo de fraturas com associação dos minerais actinolita, Fe-hornblenda actinolítica, Fe-hornblenda, albita e epidoto.

Em Adrianópolis ocorrem dois tipos de rochas máficas. $\mathrm{O}$ primeiro, de ocorrência mais restrita, é semelhante ao metagabro de Apiaí, exibindo texturas blasto subofíticas, com orto e clinopiroxênio, labradorita e actinolita metamórfica (em fraturas e interstícios). O segundo é um anfibolito que tem como constituinte principal a hornblenda, com actinolita subordinada em cristais isolados ou associados à hornblenda como manchas ou zoneamentos. Plagioclásio (oligoclásio, albita), epidoto, biotita, clorita, titanita e opacos completam a mineralogia (Maniesi, 1997; Maniesi \& Oliveira, 2000).

Já em Rio Branco do Sul, o terceiro local estudado do Grupo Açungui, os corpos de anfibolito são alongados segundo a direção NE-SW, exibem textura granoblástica e tem como mineralogia principal o anfibólio, com predomínio da actinolita, albita, epidoto, clorita e titanita. Ainda preservados do metamorfismo encontram-se clinopiroxênio e hornblenda castanha. A textura reliquiar subofítica é por vezes observada.

As ocorrências de Campo Largo estão inseridas em metassedimentos da Formação Água Clara do Grupo Setuva, nas proximidades do contato com rochas do Grupo Açungui. Constituem corpos de anfibolito alongados 
também na direção NE-SW, exibindo textura granoblástica e composição mineralógica onde se destacam o anfibólio, com predomínio da actinolita sobre a hornblenda, em cristais homogêneos ou compostos pelas duas fases em indivíduos zonados ou manchados, albita (oligoclásio é raro), quartzo, epidoto, clorita e opacos (Maniesi \& Oliveira, 2000).

Com relação à idade dessas ocorrências, as determinações radiométricas disponíveis na literatura, dizem respeito ao metagabro de Apiaí e indicam valores radiométricos neoproterozóicos de $617 \pm 4 \mathrm{Ma}$ (idade $\mathrm{U} / \mathrm{Pb}$ em zircão; Hackspacher et al., 2000) e de $612 \pm 21 \mathrm{Ma}\left(\right.$ idade $^{40} \mathrm{Ar} /{ }^{39} \mathrm{Ar}$ em plagioclásio; Allen Fetter, comunicação verbal), interpretados por esses autores como a idade de cristalização desse corpo. O metagabro de Apiaí acha-se também datado pelo método $\mathrm{Rb} / \mathrm{Sr}$ em rocha total, que forneceu uma idade de $850 \pm 85 \mathrm{Ma}$ (Daitx et al., 1990), resultado interpretado como sendo a idade de resfriamento da intrusão.

\section{RESU LTADOS E DISCUSSÃO}

\section{Idades $\mathrm{Sm} / \mathrm{Nd}$}

As idades obtidas por esta metodologia (Sato et al., 1995), referem-se a duas isócronas minerais.

A primeira isócrona foi obtida para o metagabro de Apiaí (Amostra PI 28, Tabela 1) e foi construída utilizandose dados analíticos de rocha total, plagioclásio e piroxênio, que forneceu uma idade de $885 \pm 53 \mathrm{Ma}$ (Figura 2-A), com MSWD de 0,56. A segunda foi construída para uma metabásica de Adrianópolis, com dados de rocha total, plagioclásio e piroxênio (amostra AD 27, Tabela 1), e evidenciou uma idade de $820 \pm 84 \mathrm{Ma}$ (Figura 2-B), com MSWD de 0,80 . Estes resultados são comparáveis dentro do erro, o que sugere representatividade geológica às idades obtidas, por tratar-se de ocorrências pouco distantes entre si.

Conforme já mencionado no presente texto, valor semelhante a esses foi obtido por Daitx et al. (1999), em uma isócrona $\mathrm{Rb} / \mathrm{Sr}$ de rocha total, construída com seis amostras cogenéticas do metagabro de Apiaí. Esta isócrona foi aqui recalculada, usando o programa Isoplot de Ludwig (1999), a partir dos dados constantes da Tabela 2 e o resultado está apresentado na Figura 3. A idade obtida é de $839 \pm 85$ Ma, com razão inicial 0,70499 $\pm 0,00027$, novamente concorda, dentro do erro, com os valores radiométricos obtidos pela metodologia $\mathrm{Sm} / \mathrm{Nd}$.

Assim, com base nas idades neoproterozóicas existentes $(617 \pm 4$ Ma e $612 \pm 21 \mathrm{Ma})$ para o metagabro de Apiaí, tidas como a idade de cristalização desse corpo, os valores ora determinados (Figuras 2-A, 2-B e 3) para as rochas de Apiaí e Adrianópolis, possivelmente representam aépoca de extração do manto do magma que gerou os corpos metabásicos, já que idades $\mathrm{T}_{\mathrm{DM}}$, especificamente para essas amostras não puderam ser calculadas em função dos resultados positivos de $\varepsilon_{\mathrm{Nd}}$ (Tabela 1 ).

Finalmente, a Tabela 1 apresenta as idades modelo calculadas para as amostras com os maiores valores de $\varepsilon_{\mathrm{Nd}}$ negativos (quatro amostras). Tentativamente foram calculados $\mathrm{T}_{\mathrm{DM}}$ para essas amostras, e as idades variam entre 2163 e $2823 \mathrm{Ma}$. As demais amostras, por apresentarem valores de $\varepsilon_{\mathrm{Nd}(0)}$ positivos, não permitem o cálculo da idade para o modelo $\mathrm{T}_{\mathrm{DM}}$. De todo modo, pela variação dos valores de $\mathrm{T}_{\mathrm{DM}}$ calculados, sugere-se a presença de processos de contaminação crustal nessas amostras.

Tabela 1. Resultados das determinações Sm e N d para as amostras de Adrianópolis (AD), Campo Largo (CL), Rio Branco do Sul (RB) e Apiaí (PI). $\mathcal{E}_{(\mathrm{T} 1)}=620 \mathrm{Ma}$ e $\mathcal{E}_{(\mathrm{T} 2)}=850 \mathrm{Ma}(\mathbf{R T}=$ rocha total, px = piroxênio e pl = plagioclásio)

\begin{tabular}{|c|c|c|c|c|c|c|c|c|c|c|c|}
\hline AMOSTRA & MATERIAL & Sm (ppm) & Nd (ppm) & ${ }^{147} \mathrm{Sm} /{ }^{144} \mathrm{Nd}$ & \pm Erro & ${ }^{143} \mathrm{Nd} /{ }^{144} \mathrm{Nd}$ & \pm Erro & Тум Ма $\varepsilon_{0}$ & \multicolumn{2}{|c|}{$\varepsilon_{(\mathrm{T} 1)}$} & $\varepsilon_{(\mathrm{T} 2)}$ \\
\hline AD-8A & Anfibolito/RT & 4.866 & 20.876 & 0.1409 & \pm 0.0005 & 0.512012 & \pm 0.000011 & $2163 \pm 24$ & $-12,21$ & $-7,84$ & $-6,17$ \\
\hline AD-23A & Anfibolito/RT & 3.157 & 11.919 & 0.1601 & \pm 0.0005 & 0.512072 & \pm 0.000011 & $2806 \pm 35$ & $-11,04$ & $-8,17$ & $-7,08$ \\
\hline AD-26 & Metabásica/RT & 2.010 & 4.965 & 0.2448 & \pm 0.0009 & 0.513036 & \pm 0.000025 & & 7,76 & 3,99 & 2,55 \\
\hline AD-27 & Metabásica/RT & 1.646 & 3.825 & 0.2601 & \pm 0.0012 & 0.513132 & \pm 0.000021 & & 9,64 & 4,16 & 2,76 \\
\hline AD-27 & Metabásica/px & 1.868 & 4.052 & 0.2788 & \pm 0.0009 & 0.513209 & \pm 0.000014 & & & & \\
\hline AD-27 & Metabásica/pl & 0.230 & 0.872 & 0.1594 & \pm 0.0050 & 0.512569 & \pm 0.000030 & & & & \\
\hline CL-4 & Anfibolito/RT & 2.286 & 7.061 & 0.1958 & \pm 0.0007 & 0.512704 & \pm 0.000022 & & 1,29 & 1,36 & 1,39 \\
\hline CL-11 & Anfibolito/RT & 1.766 & 5.036 & 0.2121 & \pm 0.0007 & 0.512895 & \pm 0.000014 & & 5,01 & 3,81 & 3,35 \\
\hline RB-11.7 & Anfibolito/RT & 0.848 & 2.530 & 0.2026 & \pm 0.0007 & 0.512882 & \pm 0.000014 & & 4,76 & 4,29 & 4,12 \\
\hline RB-12 & Anfibolito/RT & 3.621 & 11.077 & 0.1977 & \pm 0.0007 & 0.512721 & \pm 0.000014 & & 1,62 & 1,54 & 1,51 \\
\hline PI-13 & Metagabro/RT & 3.601 & 12.237 & 0.1779 & \pm 0.0006 & 0.512477 & \pm 0.000010 & $2431 \pm 46$ & $-3,14$ & $-1,67$ & $-1,11$ \\
\hline PI-28 & Metagabro/RT & 1.850 & 6.110 & 0.1831 & \pm 0.0006 & 0.512490 & \pm 0.000010 & $2823 \pm 56$ & $-2,89$ & $-1,82$ & $-1,41$ \\
\hline PI-28 & Metabásica/px & 2.992 & 8.769 & 0.2063 & \pm 0.0007 & 0.512635 & \pm 0.000008 & & & & \\
\hline PI-28 & Metabásica/pl & 0.262 & 1.550 & 0.1022 & \pm 0.0003 & 0.512030 & \pm 0.000016 & & & & \\
\hline
\end{tabular}



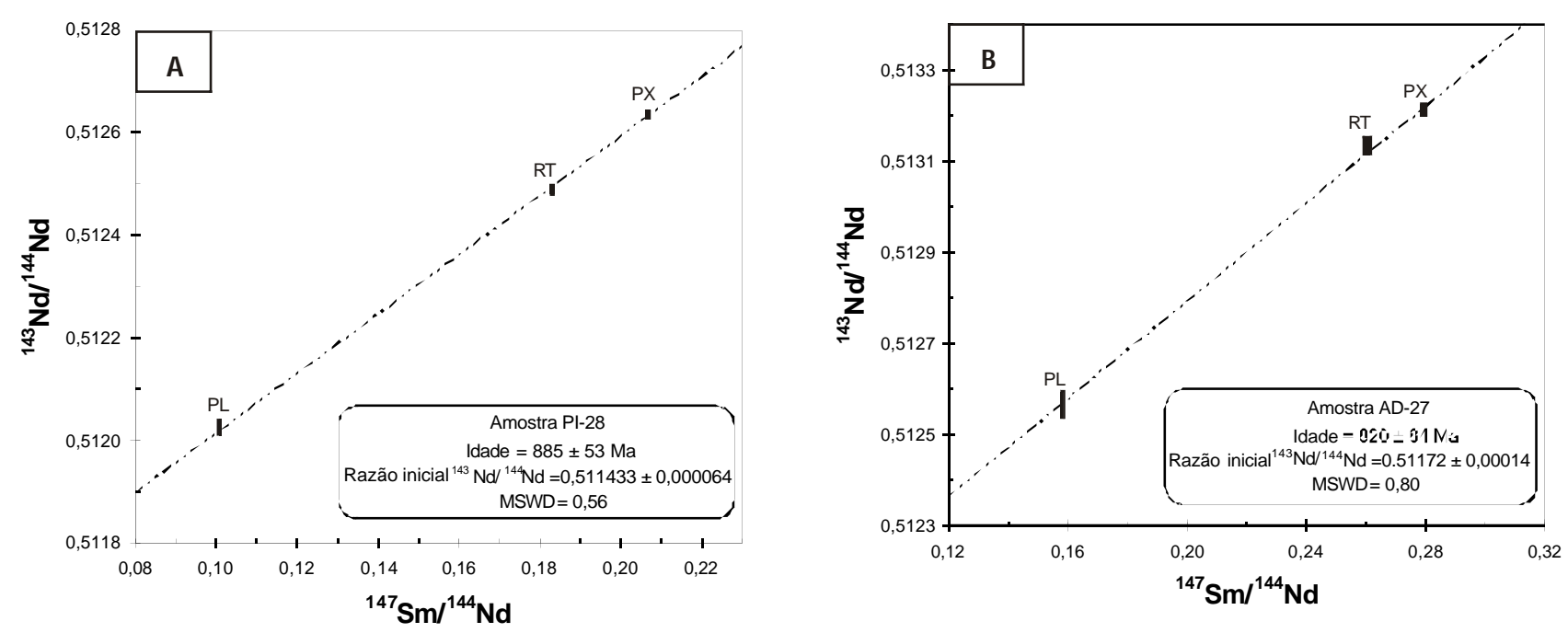

Figura 2. Diagramas isocrônicos mineral $\mathrm{Sm} / \mathrm{Nd}$ para o metagabro de Apiaí (A) e a metabásica de Adrianópolis (B), com cálculos baseados no modelo de Ludwig, 1999. Foram utilizados rocha total (RT), plagioclásio (PL) e piroxênio (PX) das a mostras analisadas.

Tabela 2. Resultados das razões $\mathrm{Rb} / \mathrm{Sr}$ e $\mathrm{Sr} / \mathrm{Sr}$ para o metagabro de Apiaí, recalculados de Daitx et al., 1990.

\begin{tabular}{llcr} 
Num. Lab. & Amostra & ${ }^{87} \mathbf{R b} /{ }^{\mathbf{8}} \mathbf{S r} \pm$ erro & ${ }^{87} \mathbf{S r} \mathbf{~}^{\mathbf{8 6}} \mathbf{S r} \pm$ erro \\
\hline 8559 & ED-07A & $0,126 \pm 0,001$ & $0,70669 \pm 0,00060$ \\
8560 & ED-20 & $0,260 \pm 0,002$ & $0,70835 \pm 0,00080$ \\
8561 & ED-01B & $0,325 \pm 0,004$ & $0,70889 \pm 0,00023$ \\
8562 & ED-01A & $0,367 \pm 0,005$ & $0,70931 \pm 0,00075$ \\
8563 & ED-07B & $0,122 \pm 0,001$ & $0,70622 \pm 0,00031$ \\
8564 & ED-02D & $0,094 \pm 0,001$ & $0,70619 \pm 0,00022$
\end{tabular}

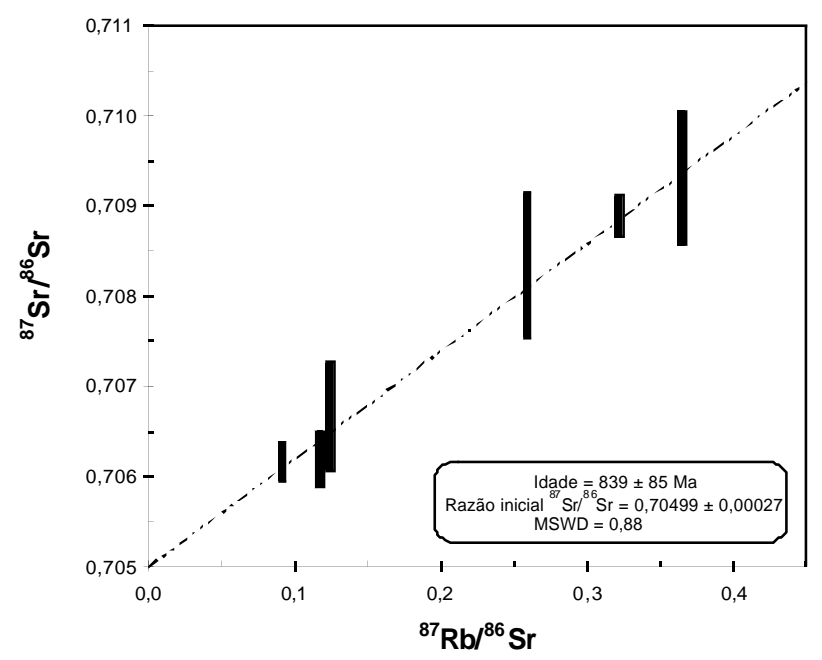

Figura 3. Diagrama isocrônico $\mathrm{Rb} / \mathrm{Sr}$ para amostras do metagabro de Apiaí constantes da Tabela 2, recalculado de Daitx etal. (1990), segundo o modelo de Ludwig (1999). 


\section{Resultados Isotópicos de $\mathrm{Pb}, \mathrm{Sr}$ e $\mathrm{Nd}$}

Os dados apresentados baseiam-se em medidas realizadas para isótopos de $\mathrm{Pb}, \mathrm{Rb}, \mathrm{Sr}, \mathrm{Sm}$ e $\mathrm{Nd}$, feitas em dez amostras, sendo quatro de Adrianópolis e duas de Apiaí, Campo Largo e Rio Branco do Sul, envolvendo os Grupos Açungui e Setuva.

Para $\mathrm{Pb}$ foram determinados os valores das razões ${ }^{206} \mathrm{~Pb} /{ }^{204} \mathrm{~Pb},{ }^{207} \mathrm{~Pb} /{ }^{204} \mathrm{~Pb}$ e ${ }^{208} \mathrm{~Pb} /{ }^{204} \mathrm{~Pb}$ para as 10 amostras, indicadas pelas siglas AD - Adrianópolis, CL - Campo Largo, RB - Rio Branco do Sul e PI - Apiaí. A ocorrência de Adrianópolis está representada por quatro análises, sendo duas do anfibolito e duas da metabásica. Os resultados estão apresentados na Tabela 3, não sendo verificados valores discrepantes.

Os valores de ${ }^{207} \mathrm{~Pb} / 206 \mathrm{~Pb}$, quando projetados em diagramas que tem como base a razão ${ }^{206} \mathrm{~Pb} /{ }^{204} \mathrm{~Pb}$ e, onde estão plotados os campos do MORB, manto depletado, manto enriquecido I e II e a crosta continental superior e inferior (Rollinson, 1993), mostram uma tendência semelhante ao alinhamento geral desses diversos campos isotópicos, onde as amostras relativamente mais evoluídas são as do anfibolito de Adrianópolis e as mais primitivas as de Campo Largo e de Rio Branco do Sul. Os pontos do metagabro de Apiaí agrupam-se com as amostras de Adrianópolis. Algumas amostras sugerem ter passado por contaminação crustal, pois se localizam, nesse diagrama, no campo da crosta continental inferior. São elas: AD 8A e 23A, anfibolitos de Adrianópolis e as duas amostras do metagabro de Apiaí: PI 13 e PI 28 (Figura 4).

Tabela 3. Razões isotópicas de Pb (composições corrigidas para balanço de massa).

\begin{tabular}{|c|c|c|c|c|c|c|}
\hline AMOSTRA & ${ }^{206} \mathrm{~Pb} /{ }^{204} \mathrm{~Pb}$ & \pm Erro $(\%)$ & ${ }^{207} \mathrm{~Pb} /{ }^{204} \mathrm{~Pb}$ & \pm Erro $(\%)$ & ${ }^{208} \mathrm{~Pb} /{ }^{204} \mathrm{~Pb}$ & $\pm \operatorname{Erro}(\%)$ \\
\hline AD-8A & 18.035 & \pm 0.010 & 15.585 & \pm 0.010 & 38.346 & \pm 0.010 \\
\hline AD-23A & 18.193 & \pm 0.022 & 15.606 & \pm 0.022 & 38.554 & \pm 0.024 \\
\hline AD-26 & 17.235 & \pm 0.083 & 15.554 & \pm 0.061 & 37.957 & \pm 0.074 \\
\hline AD-27 & 17.596 & \pm 0.015 & 15.602 & \pm 0.015 & 38.068 & \pm 0.014 \\
\hline CL-4 & 16.925 & \pm 0.027 & 15.510 & \pm 0.023 & 36.956 & \pm 0.023 \\
\hline CL-11 & 16.796 & \pm 0.020 & 15.544 & \pm 0.022 & 36.972 & \pm 0.022 \\
\hline RB-11.7 & 17.421 & \pm 0.040 & 15.557 & \pm 0.042 & 37.177 & \pm 0.043 \\
\hline RB-12 & 16.944 & \pm 0.011 & 15.557 & \pm 0.012 & 36.837 & \pm 0.013 \\
\hline PI-13 & 17.620 & \pm 0.037 & 15.516 & \pm 0.011 & 38.445 & \pm 0.012 \\
\hline PI-28 & 17.562 & \pm 0.009 & 15.502 & \pm 0.008 & 38.570 & \pm 0.010 \\
\hline
\end{tabular}

Figura 4. Diagrama ${ }^{207} \mathrm{~Pb} /{ }^{204} \mathrm{~Pb}$ versus ${ }^{206} \mathrm{~Pb} /{ }^{204} \mathrm{~Pb}$ para os metabasitos $(\backslash)$ e anfibolitos $(\boldsymbol{\Lambda})$ de Adrianópolis, e para os anfibolitos de Campo Largo (+), Rio Branco do Sul $\nleftarrow$ ) e Apiaí ( ). Limites para os diversos ambientes petrológicos segundo Rollinson (1993).

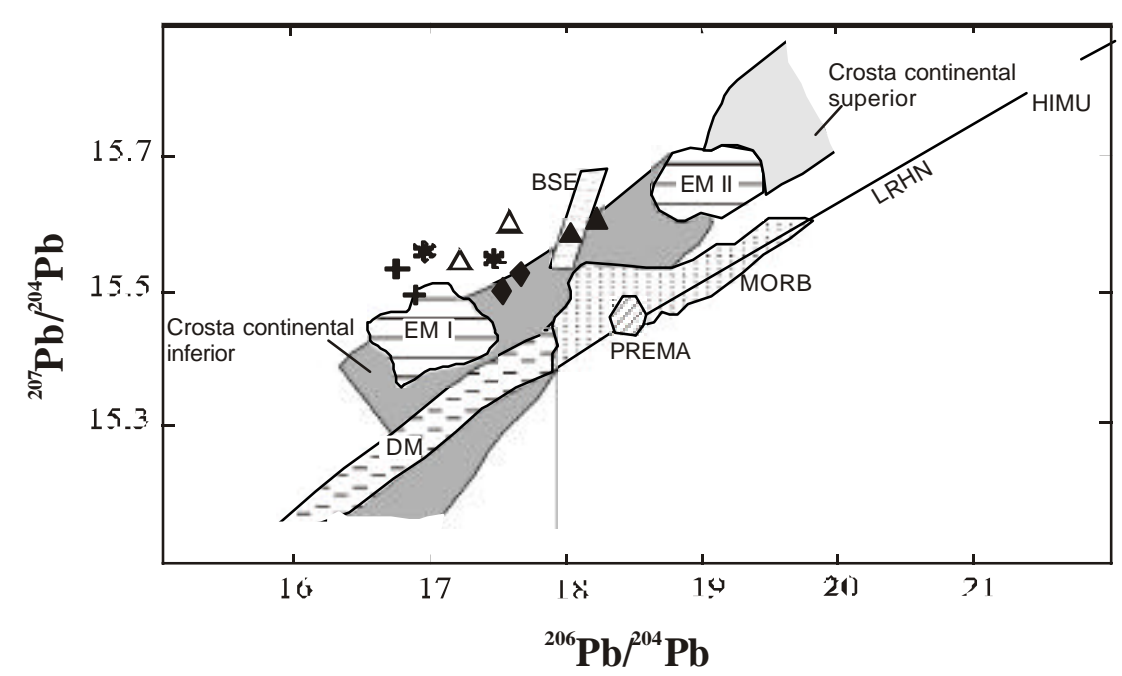


Em se considerando as razões ${ }^{87} \mathrm{Sr} /{ }^{86} \mathrm{Sr}$ (Tabelas 2 e 4), recalculadas para $\mathrm{T}=620 \mathrm{Ma}$, idade determinada por Hackspacher et al. (2000) e para T = $850 \mathrm{Ma}$, valor médio aqui assumido, evidencia-se que, ao lado de alguns valores considerados normais para essas rochas nas épocas consideradas como 0,70157 para a amostra AD 26A, aparecem outros muito reduzidos como 0,69971 para a amostra $\mathrm{AD} 26 \mathrm{~A}$, indicativo de perda de $\mathrm{Rb}$, alem de outros muito elevados como 0,70716, para a amostra AD 8A, 0,70956, para amostra RB 12 e até 0,71608, para a amostra CL 04. Para o metagabro de Apiaí um valor de 0,70499 $\pm 0,00027$ foi obtido para a razão inicial ${ }^{87} \mathrm{Sr} /{ }^{86} \mathrm{Sr}$, para a idade de $839 \pm 85 \mathrm{Ma}$ (Figura 3). Esses resultados parecem refletir a existência de contaminação crustal ou modificações causadas pelo metamorfismo em pelo menos parte das amostras ora estudadas.

Considerando as idades neoproterozóicas determinadas para as rochas de Apiaí (Hackspacher et al., 2000), foi calculado o $\varepsilon_{\mathrm{Nd}-620}$, Tabela 4. Calculou-se também o $\varepsilon_{\mathrm{Nd}}$ para o valor médio de $850 \mathrm{Ma}$, pois esse valor está sendo interpretado, possivelmente como a idade de extração do manto do líquido gerador das rochas de Apiaí e Adrianópolis.

Os valores de $\varepsilon_{\mathrm{Nd}(850)}$, obtidos para rochas totais, foram respectivamente $+2,76$ e $+2,55$ para Adrianópolis (amostras AD 27 e AD 26) e -1,41 e -1,11 para Apiaí (amostras PI 28 e PI 13). O valor para o manto depletado, para essa idade ( $850 \mathrm{Ma}$ ) é de $+6,05$, o que não invalida a hipótese inicial, considerando que o conjunto de amostras evidencia graus variados de contaminação crustal.

O exame da Tabela 1 revela que os valores de $\varepsilon_{0}$ estão agrupados em três conjuntos: o primeiro para as amostras com intervalo entre $-11,04$ e $-12,21$, o segundo para as amostras com intervalo entre $-2,85$ e $-3,14$ e ainda um terceiro para as amostras com intervalo entre $+9,64 \mathrm{e}+1,29$. Isto indica trajetórias evolutivas diferentes decorrentes de gêneses distintas ou de processos de contaminação crustal. Fato semelhante é observado quando se analisam os resultados para $620 \mathrm{Ma}\left(\varepsilon_{\mathrm{T} 1}\right)$. Assim, parâmetros negativos foram obtidos para os anfibolitos de Adrianópolis, amostras $\operatorname{AD} 8 \mathrm{~A}(-7,84)$ e amostra $\operatorname{AD} 23 \mathrm{~A}(-8,17)$ e para o metagabro de Apiaí, amostras PI $13(-1,67)$ e PI $28(-1,82)$, corroborando, portanto a hipótese de contaminação crustal e/ou de modificações metamórficas. As demais ocorrências de Adrianópolis, Campo Largo e Rio Branco do Sul exibem valores de $\varepsilon_{620}$ positivos variando entre $+4,29$ (amostra RB 11,7) e +1,36 (amostra CL 4), que se devem a fracionamento isotópico particular desses sistemas. Nos casos dos parâmetros negativos calculados, reforça-se novamente a possibilidade de contaminação crustal, no tocante ao anfibolito de Adrianópolis e amostras do metagabro de Apiaí. Isto confirma a suposição levantada a partir dos resultados dos isótopos de $\mathrm{Pb}$ de que processos de interação com a crosta afetaram, pelo menos em parte, o material estudado (Figura 4).

$\mathrm{Na}$ tentativa de correlacionar os valores de ${ }^{143} \mathrm{Nd} /{ }^{144} \mathrm{Nd}\left(\varepsilon_{\mathrm{Nd}-\mathrm{t}}\right) \mathrm{e}^{87} \mathrm{Sr} /{ }^{86} \mathrm{Sr}$, foi construída a Figura 5, onde estão delimitados os campos do MORB, OIB, do basalto continental e rift continental, e de basalto de arco de ilhas e margem continental ativa, além da trajetória do “mantle array" segundo De Paolo \& Wasserburg (1979), Wilson (1995) e Rollinson (1993). Observa-se que, à exceção das amostras RB 12, CL 04 e CL 11, todas as demais distribuem-se segundo uma tendência, a partir do MORB e que é a tendência próxima do "mantle array" ou do fracionamento magmático. Por outro lado observa-se que uma parte das amostras plota no quadrante IV ( $/ \mathrm{Sm}<0$ e $f \mathrm{Rb}>0$ ) da referida figura, que é indicativo da atuação de processos de contaminação, enquanto a outra parte dos pontos situa-se no quadrante I $(f \mathrm{Sm}>0$ e $f \mathrm{Rb}>0)$, evidenciando também que processos ligados ao metamorfismo ou à contaminação crustal podem ter modificado o quimismo inicial dessas rochas.

\section{CON SIDERAÇÕES FINAIS}

Os estudos isotópicos realizados a partir de diferentes técnicas, sustentados por dados geocronológicos, possibilitaram ampliar o conhecimento acerca da gênese das rochas anfibolíticas e metabásicas que ocorrem na região sudeste de São Paulo e nordeste do Paraná, na área de influência dos Grupos Açungui e Setuva. Os resultados permitem conhecer o comportamento dos principais isótopos e suas razões e traçar um caminho evolutivo mais detalhado para as rochas metabásicas dessa porção da Faixa Ribeira.

O conhecimento da evolução cronológica do metagabro de Apiaí, bem como da metabásica de Adrianópolis, está mais completo, pois o valor médio de $850 \mathrm{Ma}$, obtido a partir de isócronas minerais $\mathrm{Sm} / \mathrm{Nd}$, aparentemente apontam para a idade de extração do líquido magmático a partir de um manto já enriquecido, já que a idade de colocação (cristalização) é aqui assumida como sendo a da idade $\mathrm{U} / \mathrm{Pb}$ de $617 \pm 4 \mathrm{Ma}$ (Hackspacher et al., 2000).

As características isotópicas das quatro ocorrências estudadas confirmam a conclusão proveniente da geoquímica de que, cada uma delas, provem de fonte mantélica diferente, sendo a metabásica de Adrianópolis a 
Tabela 4. Resultados das determinações Rb e Sr para as amostras de Adrianópolis (AD), Campo Largo (CL), Rio Branco do Sul (RB) e Apiaí (PI).

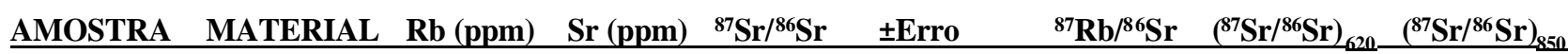

$\begin{array}{llrrrrrrr}\text { AD-8A } & \text { Anfibolito } & 30 & 205 & 0.71230 & \pm 0.000020 & 0.424 & 0.70856 & 0.70716 \\ \text { AD-23A } & \text { Anfibolito } & 14 & 211 & 0.70792 & \pm 0.000057 & 0.192 & 0.70622 & 0.70559 \\ \text { AD-26 } & \text { Metabásica } & 7 & 88 & 0.70437 & \pm 0.000021 & 0.230 & 0.70233 & 0.70157 \\ \text { AD-27 } & \text { Metabásica } & 10 & 67 & 0.70495 & \pm 0.000120 & 0.432 & 0.70113 & 0.69971 \\ \text { CL-4 } & \text { Anfibolito } & 16 & 99 & 0.72177 & \pm 0.000065 & 0.435 & 0.71763 & 0.71608 \\ \text { CL-11 } & \text { Anfibolito } & - & - & 0.72066 & \pm 0.000022 & - & - & - \\ \text { RB-11.7 } & \text { Anfibolito } & 7 & 81 & 0.70497 & \pm 0.000049 & 0.250 & 0.70276 & 0.70193 \\ \text { RB-12 } & \text { Anfibolito } & 12 & 89 & 0.71431 & \pm 0.000029 & 0.391 & 0.71085 & 0.70956 \\ \text { PI-13 } & \text { Metabásica } & 7 & 154 & 0.70666 & \pm 0.000028 & 0.132 & 0.70550 & 0.70506 \\ \text { PI-28 } & \text { Metabásica } & 3 & 131 & 0.70703 & \pm 0.000071 & 0.055 & 0.70703 & 0.70703\end{array}$

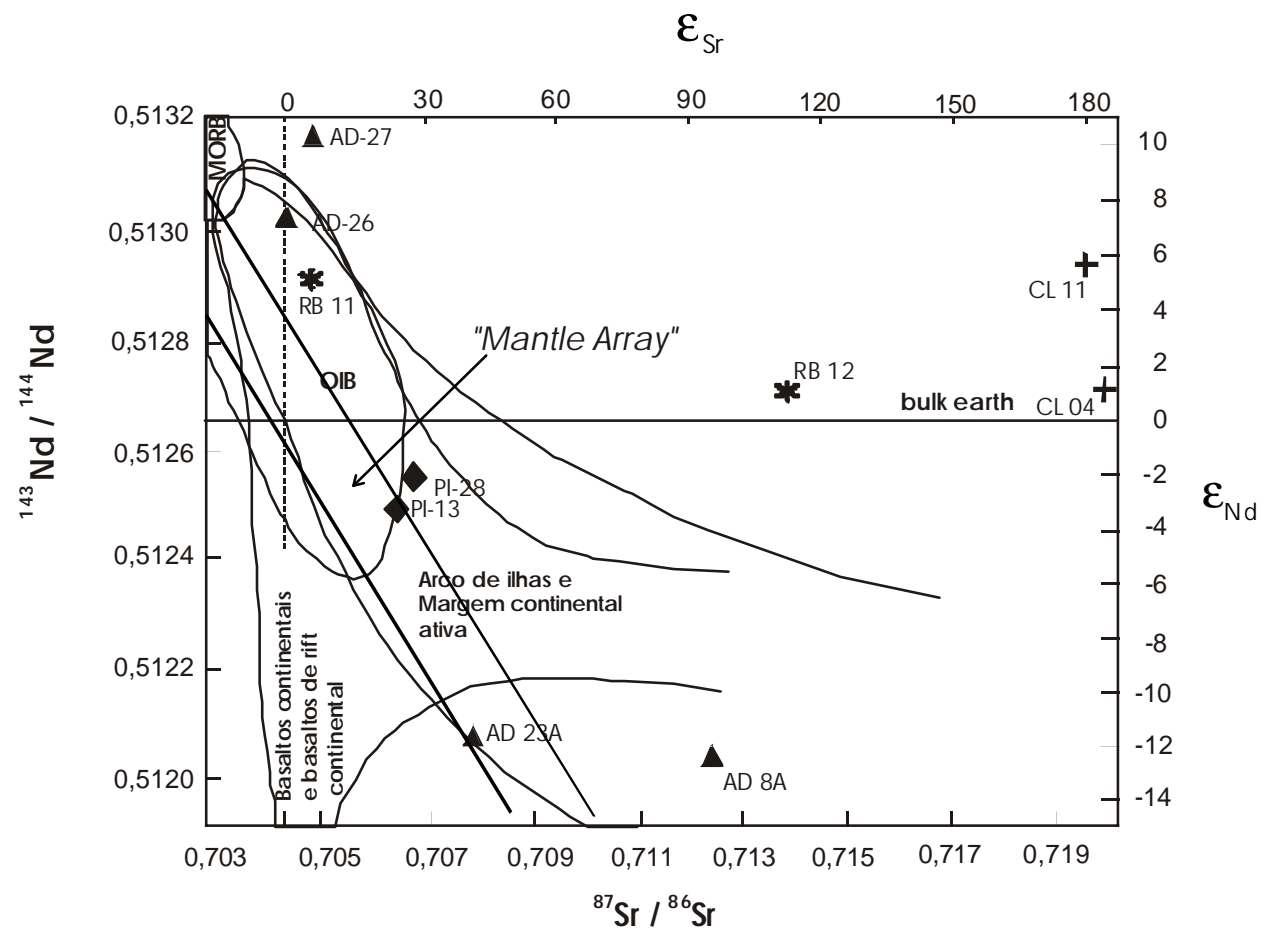

Figura 5. Diagrama de variação $\left({ }^{143} \mathrm{Nd} /{ }^{144} \mathrm{Nd}\right)_{0}$ versus $\left({ }^{87} \mathrm{Sr} /{ }^{86} \mathrm{SR}\right)_{0}$ para os metabasitos de Adrianópolis (AD), Campo Largo $(C L)$, Rio Branco do Sul (RB) a Apiaí (PI), com delimitação dos campos para diversos ambientes petrológicos segundo De Paolo $\&$ Wasserburg (1979); Wilson(1995) e Rollinson (1993).

mais primitiva, mais próxima do campo isotópico do DM (depleted mantle, Taylor \& McLennan, 1985) com a razão ${ }^{87} \mathrm{Sr} /{ }^{86} \mathrm{Sr} \pm 0,7046$ e $\varepsilon_{\mathrm{Nd}-0}+8$ em média e os anfibolitos, também de Adrianópolis, as mais evoluídas e que mais se aproximam do campo isotópico do manto enriquecido $\left({ }^{87} \mathrm{Sr} /{ }^{86} \mathrm{Sr}\right.$ entre 0,7079 e 0,$7046 ; \mathcal{E}_{\mathrm{Nd}-0}$ entre-11,04 e-12,21), sendo que, no geral, elas seguem a tendência do "mantle array". Para os anfibolitos é preciso lembrar, no entanto, que processos metamórficos podem ser responsáveis pe- los elevados valores negativos de $\varepsilon_{\mathrm{Nd}(0)}$, pois o metamorfismo deve ter provocado modificações químicas nessas rochas.

Com base nos resultados, pode-se concluir, por outro lado, que processos de contaminação crustal afetaram, parte desses materiais, de maneira variada. Assim, observa-se contaminação em isótopos de $\mathrm{Pb}$ para os anfibolitos de Adrianópolis e para o metagabro de Apiaí. Também estão contaminadas, conforme indicado pelas elevadas razões 
$\mathrm{Sr}^{87} / \mathrm{Sr}^{86}$, uma amostra de Rio Branco do Sul (RB 12), duas de Campo Largo (CL 04 e 11) e uma de Adrianópolis, o anfibolito AD 8A, sendo que a amostra AD 27 apresenta perda de $\mathrm{Rb}$, indicada pelo valor da razão ${ }^{87} \mathrm{Sr} /{ }^{86} \mathrm{Sr}$ de 0,69971, para a idade de 850 Ma. É possível assim, propor que as anomalias de $\mathrm{Rb}, \mathrm{Ba}, \mathrm{Nb}$, Th e $\mathrm{Sm}$, apontadas por estudos geoquímicos de Maniesi (1997) e Maniesi \& Oliveira (2000), resultem possivelmente de processos de contaminação crustal.

Para os corpos de Rio Branco do Sul e Campo Largo, com valores de $\varepsilon_{\mathrm{Nd}}$ positivos pode-se confirmar sua origem mantélica, já indicada pelos dados geoquímicos. Para Campo Largo, no entanto, os elevados resultados da razão ${ }^{87} \mathrm{Sr} /{ }^{86} \mathrm{Sr}$, mesmo quando considerados para valores de T de 620 Ma e $850 \mathrm{Ma}$, são indicativos de forte contaminação crustal desses anfibolitos

Para finalizar, considerando que os dados ora apresentados são preliminares, torna-se evidente que mais determinações são necessárias para confirmar as hipóteses ora levantadas, considerando os processos de contaminação e metamorfismo que envolveram essas rochas, o que demandaria um número maior de medidas isotópicas para cada um dos corpos de metabásicas e anfibolitos aqui estudados.

\section{AG RADECIMENTOS}

Os autores agradecem à FAPESP pelo suporte financeiro (Processo ${ }^{\circ}$ 97/12458-7) e aos revisores desta revista pelas sugestões e críticas ao manuscrito.

\section{REFERÊN CIAS BIBLIO GRÁFICAS}

ALMEIDA, F. F. M. (1956) Novas ocorrências de fósseis no pré-cambriano brasileiro. Anais da Academia Brasileira de Ciências, v. 28, n. 4, p. 44-45.

ALMEIDA, F. F. M. de; AMARAL, G.; CORDANI, U. G.; KAWASHITA, K. (1973) The precambrian evolution of the South American cratonic margin south of Amazon River. In: NAIRN, E, M.; STEHLI, F. G. (eds.) The ocean basins and margins. New York, Plenum. v. 1, p. 411-446.

BIGARELLA, J. J.; SALAMUNI, R. (1956) Estudos preliminares da Série Açungui V - estruturas organógenas nos dolomitos da Formação Capiru (PR). Dusenia, v. 7 , n. 6 , p. 317-323.
CAMPANHA, G. A. C. (1991) Tectônica proterozóica do alto e médio vale do Ribeira, estados de São Paulo $e$ Paraná. São Paulo, 296 p. Tese (Doutorado) - Instituto de Geociências, Universidade de São Paulo.

CAMPANHA, G. A. C.; BISTRICHI, C. A.; ALMEIDA, M. A. (1987) Considerações sobre a organização litoestratigráfica e evolução tectônica da faixa de dobramentos Apiaí. In: SIMPÓSIO SUL-BRASILEIRO DE GEOLOGIA, 3., Curitiba, 1987. Atas. Sociedade Brasileira de Geologia. v. 2, p. 725-742.

CAMPANHA, G. A. C.; SADOWSKI, G. R. (1999) Tectonics of the Southern portion of the Ribeira Belt (Apiaí Damain). Precambrian Research, v. 98, p. 31-51.

CAMPOS NETO, M. C.; FIGUEIREDO, M. C. H. (1995) The Rio Doce Orogeny, Southeastern Brazil.Journal of South American Earth Sciences, v. 8, n. 2. p. 143-162.

DAITX, E. C.; TEIXEIRA, W.; ZANARDO, A. (1990) Geologia e geocronologia do metagabro de Apiaí, Vale do Ribeira, SP. In: CONGRESSO BRASILEIRO de GEOLOGIA, 36., Natal, 1990. Boletim de Resumos. Natal, Sociedade Brasileira de Geologia. p. 341.

EBERT, H. (1971) Observações sobre a litologia e subdivisão do "Grupo Setuva" noestado do Paraná, com sugestões à tectônica geral do "Geossinclínio Açungui”. In: CONGRESSO BRASILEIRO DE GEOLOGIA, 25., São Paulo. Anais. São Paulo, SBG. v. 1, p. 131-146.

DE PAOLO, D. J.; WASSERBURGG. J. (1979) Petrogenetic mixing models and $\mathrm{Nd}-\mathrm{Sr}$ isotopic patterns. Geochimica et Cosmochimica Acta, v. 43, p. 615-627.

FIORI, A.P. (1985)Estudos geológicos integrados do PréCambriano paranaense. Curitiba, UFPR/ MINEROPAR. 192p.

FIORI, A. P. (1987). Aspectos estruturais e estratigráficos do Grupo Açungui e Formação Itaiacoca no estado do Paraná. Curitiba, UFPR/MINEROPAR. 191p.

FIORI, A. P. (1990) Tectônica e estratigrafia do Grupo Açungui a norte de Curitiba. São Paulo, 191p. Tese (Livre-docência) - Instituto de Geociências, Universidade de São Paulo.

FIORI, A. P. (1993) O sistema de cavalgamento Açungui (PR). Geociências. São Paulo, v. 12, n.1, p. 187-208.

FRITZSONS JUNIOR, O.; PIEKARZ, G. F.; FLACADE, D. (1982). Geologia e potencial econômico do Grupo Setuva, PR. In: CONGRESSO BRASILEIRO DE GEOLOGIA, 32., Salvador, 1982. Anais. Salvador, 
Sociedade Brasileira de Geologia. v. 3, p. 987-1001.

HACKSPACHER, P. C.; DANTAS, E. L.; SPOLADORE, A.; FETTER, A. H.; OLIVEIRA, M. A. F. (2000) Evidence of neoproterozoic backarc basin development in the central Ribeira Belt, Southeastern, Brazil: new geochronological and geochemical constraints from the São Roque-Açungui Groups. Revista Brasileira de Geociências, v. 30, n.1, p. 110-114.

HASUI, Y.; CARNEIRO, C. D. R.; COIMBRA, A. M. (1975) The Ribeira folded belt. Revista Brasileira de Geociências, v. 5, n. 4, p. 247-266.

HASUI, Y.; CARNEIRO, C. D. R.; BISTRICHI, C. A. (1980) Estruturas e tectônica do Pré-Cambriano de São Paulo e Paraná. Anais da Academia Brasileira de Ciências, v. 52, p. 61-76.

HASUI, Y.; EBERT, H. D.; QUADE, H. (1984) Aspectos geológicos da megantiforma da Serra do Cadeado, PR. In: CONGRESSO BRASILEIRO DE GEOLOGIA, 33., Rio de Janeiro. 1984. Anais. Belo Horizonte, Sociedade Brasileira de Geologia, v. 7, p. 2380-2394.

LUDWIG, K. R. (1999) Isoplot: a plotting and regression program for radiogenic isotope data, for IBM-PC compatible computer, version $2.10 \mathrm{~b}$.

MANIESI, V. (1997) Petrologia das rochas anfibolíticas das regiões de Adrianópolis, Campo Largo e Rio Branco do Sul, PR. Rio Claro, 215p. Tese (Doutorado) - Instituto de Geociências e Ciências Exatas, Universidade Estadual Paulista.

MANIESI, V.; OLIVEIRA, M. A. F. (2000) Petrogênese dos metabasitos com afinidades dos toleitos de fundo oceânico das regiões de Adrianópolis e Campo Largo, Pr. Revista Brasileira de Geociências, v. 30 n. 4, p. 607-614.

MANIESI, V.; OLIVEIRA, M. A. F. (2002) Metabasitos com afinidades de basaltos komatiíticos da região de Rio Branco do Sul, PR. Revista Brasileira de Geociências, v. 32 n. 2, p. 147-153.

MANIESI, V.; OLIVEIRA, M. A. F.; ZANARDO, A. (1999) Petrologia da metagabro de Apiaí - dados preliminares. In: SIMPÓSIO DE GEOLOGIA DO SUDESTE, 6. São Pedro, 1999. Boletim de Resumos. São Pedro, Sociedade Brasileira de Geologia. p. 36.

PIEKARZ, G. F. (1981) Reconhecimento das unidades correlacionáveis à seqüência mineralizada do Perau, estado do Paraná. In: SIMPÓSIO REGIONAL DE GEOLOGIA, 3., Curitiba, 1981. Anais. Curitiba, Sociedade Brasileira de Geologia.v.1 p. 148-154.

PONTES, J. B. (1982) Geologia e potencialidade econômica da Formação Água Clara (PR). In: CONGRESSO BRASILEIRO DE GEOLOGIA, 32., Salvador, 1982. Anais. Salvador, Sociedade Brasileira de Geologia. v. 1, p. 1002-1016.

REIS NETO, J. M.; SOARES, P. C. (1987) Um estudo de caracterização termodinâmica de micro estruturas dos grupos Açungui e Setuva (PR). In: SIMPÓSIO SUL BRASILEIRO DE GEOLOGIA, 3., Curitiba, 1987.Anais. Curitiba, Sociedade Brasileira de Geologia. v. 1, p. $147-165$.

ROLLINSON, H. (1993) Using geochemical data: evaluation, presentation, interpretation. Longman, Londres. $351 \mathrm{p}$.

SATO, K.; TASSINARI, C. C. G.; KAWASHITA, K.; PETRONILHO, L. (1995) O método Sm-Nd no IG/USP e suas aplicações. Anais da Academia Brasileira de Ciências, v. 67, n. 3, p. 313-336.

SORES, P. C. (1987) Seqüências tectono-sedimentares e tectônica deformacional no centro oeste do escudo paranaense. In: SIMPÓSIO SUL BRASILEIRO DE GEOLOGIA, 3., Curitiba. Anais. Curitiba, Sociedade Brasileira de Geologia. v. 2, p. 743-772.

SOARES, P. C. (1988) Tectônica colisional em torno do Bloco Paraná, Brasil. In: CONGRESSO LATINO AMERICANO DE GEOLOGIA, 7., Belém, 1988. Anais. Belém, Sociedade Brasileira de Geologia. v. 1, p. 63-81

TAYLOR, S. R.; McLENNAN, S. M. (1985) The continental crust: its composition and evolution. Oxford, Blackwell. 312 p.

WILSON, M. (1995). Igneous petrogenesis. Londres, Unwin Hyman, Londres. 465p. 